\title{
Relationship between Autophosphorylation and Phosphorylation of Exogenous Substrates by the Human Cytomegalovirus UL97 Protein Kinase
}

\author{
Moon-Chang Baek, $\uparrow$ Paula M. Krosky, $\ddagger$ and Donald M. Coen* \\ Department of Biological Chemistry and Molecular Pharmacology, Harvard Medical School, \\ Boston, Massachusetts 02115
}

Received 30 May 2002/Accepted 14 August 2002

\begin{abstract}
Human cytomegalovirus encodes an unusual protein kinase, UL97, which is a member of the $\mathrm{HvU}_{\mathrm{L}}$ family of protein kinases encoded by diverse herpesviruses. UL97 is able to autophosphorylate and to phosphorylate certain exogenous substrates, including nucleoside analogs such as ganciclovir. It has previously been concluded that phosphorylation of UL97 is essential for its phosphorylation of ganciclovir. We examined the relationship between autophosphorylation of UL97 and its activity on exogenous substrates. Glutathione $S$-transferase-UL97 fusion protein purified from insect cells was found to be already partially phosphorylated, but neither extensive autophosphorylation nor phosphatase treatment meaningfully altered the time course of its phosphorylation of the exogenous substrate, histone H2B. Sequencing and mass spectrometric analyses of ${ }^{32}$ P-labeled tryptic peptides of the UL97 fusion protein identified nine sites of autophosphorylation, all within the first 200 residues of the protein, outside of conserved protein kinase subdomains. A peptide corresponding to the N-terminal UL97 segment that was most extensively autophosphorylated was readily phosphorylated by UL97, confirming that fusion protein sequences are not required for phosphorylation at this site. Deletion mutants lacking at least the first 239 residues exhibited drastically reduced autophosphorylation $(<5 \%)$ but retained near-wild-type $\mathrm{H} 2 \mathrm{~B}$ phosphorylation activity. Baculoviruses expressing these mutants efficiently directed the phosphorylation of ganciclovir in insect cells. Taken together, these results identify the autophosphorylation sites of a herpesvirus protein kinase and show that autophosphorylation of UL97 is not required for phosphorylation of exogenous substrates.
\end{abstract}

Protein kinases regulate many biological processes. Most, if not all, viruses make use of protein kinases in their life cycles, and many viruses encode their own protein kinases. Human cytomegalovirus (HCMV) encodes an unusual protein kinase known as UL97, the product of the UL97 open reading frame. UL97 is remarkable in that it can phosphorylate nucleoside analogs such as the antiviral drugs acyclovir and ganciclovir (GCV) $(12,22,23)$. Indeed, mutations that impair GCV phosphorylation by UL97 confer GCV resistance (22), which can be clinically significant (7). UL97 can also phosphorylate certain more typical protein kinase substrates such as histones (1). UL97 is important for virus replication in cell culture (18) with roles in DNA replication, DNA encapsidation and/or nuclear egress $(2,11 \mathrm{a}, 26)$.

UL97 is most closely related in sequence to certain protein kinases encoded by various herpesviruses, known as the $\mathrm{HvU}_{\mathrm{L}}$ protein kinase family $(3,6,13,20)$. These enzymes share many motifs typical of protein kinases but are relatively divergent. They are also characterized by N-terminal domains of various lengths, whose sequences exhibit little, if any conservation. The protein kinase motifs of UL97 are even more divergent than

\footnotetext{
* Corresponding author. Mailing address: Department of Biological Chemistry and Molecular Pharmacology, Harvard Medical School, 250 Longwood Ave., Boston, MA 02115. Phone: (617) 432-1691. Fax: (617) 432-3833. E-mail: Don_Coen@hms.harvard.edu.

$\dagger$ Present address: Division of Molecular and Life Sciences, Pohang University of Science and Technology, Pohang 790-784, Korea.

$\ddagger$ Present address: Screening Technologies Branch, Developmental Therapeutics Program, NCI Frederick, Frederick, MD 21702.
}

those of other family members, and it contains a particularly long (>300-residue) N-terminal domain (3).

Protein kinases often are themselves regulated by phosphorylation. Such regulatory modifications, which can occur by autophosphorylation or by an upstream enzyme, can occur within or outside the catalytic domain. These modifications can result in either activation or inhibition of enzyme activity or can mediate protein-protein interactions (9). Like many protein kinases, UL97 can autophosphorylate in vitro (10). It also is a phosphoprotein in virus-infected cells (24). In vitro, autophosphorylation occurs on Ser and Thr residues (10), but the locations of these residues within UL97 are unknown. The consequences of autophosphorylation for UL97 activity are unclear. One group of investigators found that various substitutions in motifs conserved among protein kinases impaired both autophosphorylation and the ability of viral vectors encoding these mutant forms of UL97 to direct GCV phosphorylation in infected cells (14). From these results, the investigators concluded that phosphorylation of UL97 is essential for GCV phosphorylation. However, as the mutations studied seem as likely to have impaired kinase activity generally as autophosphorylation specifically, we felt that this conclusion warranted reexamination.

To address the effects of autophosphorylation on UL97 activity, we took a two-pronged approach, making use of UL97 expressed by recombinant baculovirus as a glutathione $S$-transferase (GST) fusion protein (9). We tested the effect of enzymatically dephosphorylating or preautophosphorylating purified UL97 on its ability to phosphorylate an exogenous protein 
substrate, histone H2B. We then mapped the autophosphorylation sites of UL97, all of which lie within its N-terminal domain, and studied the effects of deleting this region of the protein on $\mathrm{H} 2 \mathrm{~B}$ phosphorylation and the ability of UL97 to direct GCV phosphorylation in recombinant baculovirus-infected insect cells. Our results show that there is no requirement that UL97 be autophosphorylated for activity on exogenous substrates.

\section{MATERIALS AND METHODS}

Cell and viruses. Spodoptera frugiperda 9 cells (Sf9), obtained from the American Type Culture Collection, were maintained in Grace's insect medium (Life Technologies) supplemented with $10 \%$ fetal bovine serum (Atlanta Biologicals), $100 \mathrm{U}$ of penicillin/ml, and $100 \mu \mathrm{g}$ of streptomycin B/ml. Recombinant baculoviruses expressing wild-type (wt) or mutant GST-UL97 proteins were propagated and maintained by standard methods (4).

Reagents. Maribavir [5,6-dichloro-2-(isopropylamino)-1- $\beta$-L-ribofuranosyl1H-benzimidazole, also known as 1263W94] and ganciclovir were kindly provided by K. K. Biron and C. Talarico, Glaxo Wellcome Co. (now Glaxo SmithKline). Unless otherwise noted, all other reagents were purchased from Sigma.

Construction of recombinant baculoviruses. Baculovirus constructs expressing wt GST-UL97 and mutant GST-UL97K355Q were reported previously (10). Baculoviruses expressing mutant GST-UL97 proteins containing truncations of $239(\Delta \mathrm{N} 239), 277(\Delta \mathrm{N} 277)$, or $303(\Delta \mathrm{N} 303)$ residues from their $\mathrm{N}$ terminus were constructed by Zuwen He of this laboratory. Briefly, oligonucleotides (5'-CCG GATCCCGGGCCCGGGCCGC-3' $\Delta$ N239, (5'-CGGGATCCACCAGATCAT CACC-3' for $\Delta \mathrm{N} 277$ ), (5'-CGGGATCCGCGAGCTCTCTATC-3' for $\Delta \mathrm{N} 303$ ) were used to amplify the corresponding fragments by PCR, by using BamHIlinearized pGSTUL97 (10) as a template and oligonucleotide 5'-ATCGTCAG TCAGTCACGA-3' as the reverse primer. The PCR fragments were cut with $B a m \mathrm{HI}$ and EcoRI and inserted into pAcG3X (PharMingen). The SphI-EcoRI, $A p a \mathrm{I}-E c o \mathrm{RI}$, and SstI-EcoRI fragments were then replaced with those of wt pGSTUL97 to ensure that these segments contained no PCR-induced mutations. DNA sequencing verified that the mutagenized segments of the plasmids were in the correct orientation and contained only the desired mutations.

Purification of baculovirus-expressed UL97 proteins. Baculovirus-expressed wt and mutant GST-UL97 proteins were purified by using a previously described method (10), modified as follows. The protein that eluted from a glutathioneSepharose column was bound to a Q-Sepharose column (Amersham Pharmacia Biotech) previously equilibrated with elution buffer (EB; $50 \mathrm{mM}$ Tris [pH 8.0], 2 $\mathrm{mM}$ dithiothreitol [DTT], $2 \mathrm{mM}$ EDTA, $10 \%$ glycerol) with $50 \mathrm{mM} \mathrm{NaCl}$ (EB50). The Q-Sepharose was washed with 30 column volumes of EB-50 and 30 column volumes of EB-150 (EB with $150 \mathrm{mM} \mathrm{NaCl}$ ), and protein was then eluted with EB-500. This material was loaded onto a phenyl-Sepharose column (Amersham Pharmacia Biotech) equilibrated with EB-1000, and the column was washed with 30 column volumes of EB-500 and 10 volumes of EB-300 with $0.1 \%$ Triton X-100. GST-UL97 was then eluted with EB-0 with $0.1 \%$ Triton X-100, concentrated with a Centricon-30, and dialyzed for $2 \mathrm{~h}$ against EB-50. This procedural modification results in protein that is apparently homogeneous based on sodium dodecyl sulfate-polyacrylamide gel electrophoresis (SDS-PAGE) and Coomassie blue staining (similar to that published previously [10]) and eliminates a contaminating protein kinase activity that was active on certain substrates. The concentrations of full-length GST-UL97 protein were determined by amino acid analysis performed by Angelo Dickerson at the Molecular Biology Core Facility (MBCF) of the Dana-Farber Cancer Institute (DFCI). For studies with truncated GST-UL97 proteins, the concentrations were quantified by the Bio-Rad microassay system relative to full-length GST-UL97 and adjusted by using the predicted molecular mass of each UL97 protein. Purified preparations were stored at $-80^{\circ} \mathrm{C}$.

Protein kinase assays. Assays of UL97 autophosphorylation were performed in kinase buffer (50 mM Tris- $\mathrm{HCl}$ [pH 9.0], $300 \mathrm{mM} \mathrm{NaCl}, 10 \mathrm{mM} \mathrm{MgCl} 2,2 \mathrm{mM}$ DTT, and $10 \mu \mathrm{M}$ ATP), with $5 \mu \mathrm{Ci}$ of $\left[\gamma^{-32} \mathrm{P}\right] \mathrm{ATP}$ at $>5,000 \mathrm{Ci} / \mathrm{mmol}(\mathrm{ICN})$ sample, unless otherwise specified, and selected concentrations of wt or mutant GST-UL97. The reactions were incubated at $37^{\circ} \mathrm{C}$ for the times specified and terminated by the addition of $6 \times$ SDS sample buffer. The samples were boiled for $3 \mathrm{~min}$ and were analyzed by SDS-PAGE and autoradiography. To assay phosphorylation of histone $\mathrm{H} 2 \mathrm{~B}, 5 \mu \mathrm{g}$ of calf thymus histone $\mathrm{H} 2 \mathrm{~B}$ (Roche) was added to the kinase reaction. To quantify autophosphorylation and $\mathrm{H} 2 \mathrm{~B}$ phosphorylation, radioactive bands were excised from dried gels, and the radioactivity was determined by liquid scintillation counting.
Effect of lambda phosphatase treatment. Wild-type GST-UL97 (800 ng) was treated with $100 \mathrm{U}$ of lambda phosphatase (NEB) for $30 \mathrm{~min}$ at $37^{\circ} \mathrm{C}$ in $20 \mu \mathrm{l}$ of the buffer supplied by the manufacturer. One-half of the reaction was then added to $40 \mu \mathrm{l}$ of kinase buffer containing $100 \mu \mathrm{M}$ orthovanadate as a phosphatase inhibitor and $100 \mu \mathrm{M}$ unlabeled ATP. The other half was added to kinase buffer containing $100 \mu \mathrm{M}$ orthovanadate without ATP. After preincubation for $30 \mathrm{~min}$ at $37^{\circ} \mathrm{C}$, the mixture without ATP was adjusted to $100 \mu \mathrm{M} \mathrm{ATP}$, and $30 \mu \mathrm{Ci}$ of $\left[\gamma^{-32} \mathrm{P}\right]$ ATP at $>5,000 \mathrm{Ci} / \mathrm{mmol}$ and histone H2B $(5 \mu \mathrm{g})$ were added to both samples. Aliquots were taken at specific times and reactions were terminated by the addition of $6 \times$ SDS sample buffer. Autophosphorylation and H2B phosphorylation were quantified as described above.

Tryptic digestion and extraction of peptide fragments. Autophosphorylated ${ }^{32}$ P-labeled GST-UL97 $(750 \mu \mathrm{g})$ was resolved by SDS-PAGE, and after the gel was dried, the labeled protein was detected by autoradiography. The band containing phosphorylated GST-UL97 was excised and placed in a microcentrifuge tube. In-gel tryptic digestion and peptide extraction were performed by a modification of a published procedure (19). To reduce the concentrations of SDS and other contaminants that can inhibit trypsin, the slices were subjected to two cycles of dehydration, rehydration, and removal of liquid. After a third dehydration, the slices were suspended in $100 \mathrm{mM}$ ammonium bicarbonate containing 50 $\mu \mathrm{g}$ of TPCK ( $p$-tosyl-L-phenylalanine chloromethyl ketone)-trypsin (sequencing grade; Roche). The phosphorylated protein was digested overnight at $37^{\circ} \mathrm{C}$. More TPCK-trypsin $(25 \mu \mathrm{g})$ was added, and incubation was continued for an additional $6 \mathrm{~h}$. Then, excess ammonium bicarbonate was removed by evaporation under vacuum. The peptides were extracted into $400 \mu \mathrm{l}$ of acetonitrile-formic acid (1:1) by intermittently vortexing for $20 \mathrm{~min}$. This step was repeated three times to optimize extraction. The extracted peptides were pooled and dried.

Alkaline gel electrophoresis (30\%) and RP-HPLC. ${ }^{32}$ P-labeled GST-UL97 tryptic peptides were loaded onto a $30 \%$ (wt/vol) alkaline acrylamide gel and electrophoresed overnight at $5 \mathrm{~mA}$, as described previously $(5,17,25)$. The radioactive bands were excised, and peptides were extracted into $0.1 \%$ trifluoroacetic acid (TFA) at $4^{\circ} \mathrm{C}$ for $3 \mathrm{~h}$ with vigorous shaking. The eluant was removed and reserved. The gel slices were further extracted overnight at $4^{\circ} \mathrm{C}$ with fresh $0.1 \%$ TFA. The two eluants were combined and dried by evaporation. Extracted peptides were dissolved in $0.1 \%$ ( $\mathrm{vol} / \mathrm{vol})$ TFA, and the solutions were adjusted to $\mathrm{pH}<2$ with $10 \%$ TFA. Reversed-phase high-performance liquid chromatography (RP-HPLC) was performed on a $\mathrm{C}_{18}$ column (Vydac 218TP52) by James Lee at the MBCF of the DFCI. The peptides were eluted with a linear gradient from 0 to $70 \%$ acetonitrile with $0.1 \%$ TFA. Fractions were collected every $30 \mathrm{~s}$, and radioactivity was monitored by Cerenkov counting.

Identification of phosphorylation sites by sequencing and mass spectrometry. The radioactive HPLC fractions were analyzed by peptide sequencing and matrix-assisted laser desorption ionization time-of-flight mass spectrometry (MALDI-TOF) by James Lee at MBCF, DFCI. Phosphopeptides were coupled to Immobilon-AA (Millipore) according to the manufacturer's instructions prior to sequencing. Sequencing was performed on an Applied Biosystems 477A system at MBCF, DFCI, by standard techniques except that $90 \%$ (vol/vol) methanol containing $15 \mathrm{ml}$ of $85 \%$ (wt/vol) phosphoric acid-100 ml was employed for residue extraction because this gave better recovery of phosphorylated derivatives. An in-line split diverted a portion of each sequencing cycle to a fraction collector to determine ${ }^{32} \mathrm{P}$ content by liquid scintillation counting, whereas the remaining portion was employed for residue identification by RP-HPLC.

Synthetic peptides. Peptides were synthesized by Research Genetics. Each peptide was verified by laser desorption mass spectroscopy for mass composition and the concentration of each solution was quantified by amino acid analysis by Angelo Dickerson at MBCF, DFCI.

Electrophoresis of phosphorylated peptides on $30 \%$ chimeric alkaline polyacrylamide gels. Peptides $(200 \mu \mathrm{M})$ were phosphorylated for $1 \mathrm{~h}$ with GST-UL97 (300 ng) in buffer containing $50 \mathrm{mM}$ Tris ( $\mathrm{pH} 9.0$ ), $20 \mathrm{mM} \mathrm{MgCl}_{2}, 5 \mathrm{mM}$ DTT, and $200 \mu \mathrm{M}$ ATP. Reactions were terminated by the addition of $6 \times$ SDS sample buffer, boiled at $100^{\circ} \mathrm{C}$ for $3 \mathrm{~min}$, and applied to a chimeric $30 \%$ alkaline acrylamide gel (1), that permits the resolution of both GST-UL97 and synthetic peptides on the same gel. In this chimeric gel system, the stacking gel is the standard 5\% stacking gel for Tris-glycine SDS-PAGE, whereas the resolving gel and running buffer are detergent free, with the same composition used for alkaline PAGE $(5,17,25)$ except that $30 \%$ acrylamide (acrylamide-bisacrylamide [30:0.037]) was used. After electrophoresis, the gels were dried and exposed to film. When appropriate, the radioactive peptides were excised, extracted, and analyzed by mass spectroscopy and amino acid sequencing as described above.

GCV anabolism and Western blotting. Sf9 cells $\left(10^{6}\right)$ in 35 -mm-diameter dishes were infected with various recombinant baculoviruses, each at a multiplicity of infection of 3 to 5 , adjusted to ensure similar levels of expression of each version of UL97. At 24 h postinfection (p.i.), the cells were pulse-labeled for 


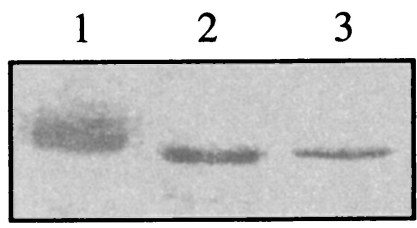

FIG. 1. Electrophoretic behavior and phosphorylation state of UL97. GST-UL97 purified from insect cells expressing either wt GSTUL97 (lanes 1 and 2) or mutant GST-UL97K355Q (lane 3) was analyzed by SDS-PAGE either after treatment with (lane 2) or without (lanes 1 and 3) lambda phosphatase.

various lengths of time with $5 \mu \mathrm{M}$ unlabeled $\mathrm{GCV}$ and $5 \mu \mathrm{Ci}$ of ${ }^{3} \mathrm{H}-\mathrm{GCV}$ (Moravek), which had been purified by HPLC to remove guanine contaminants. At various times, cells were scraped into the medium and pelleted by low-speed centrifugation. Each cell pellet was resuspended in $0.2 \mathrm{ml}$ of phosphate-buffered saline, sonicated twice for 2 min each with a cup horn sonicator, and the suspension was clarified by centrifugation for $5 \mathrm{~min}$ at $13,000 \mathrm{rpm}$ in a microfuge. Phosphorylation of GCV was assayed by using a filter-binding assay. Briefly, 50 $\mu \mathrm{l}$ of cell extract was spotted onto DE81 filters (Whatman). Filters were dried, washed three times for 5 min each with $10 \mathrm{mM}$ formic acid, once with water, and once with $90 \%$ ethanol. After drying, the filters were placed in scintillation vials and $1 \mathrm{ml}$ of $2 \mathrm{M} \mathrm{NaCl}-0.2 \mathrm{M} \mathrm{HCl}$ was added to each vial. Then, $10 \mathrm{ml}$ of scintillation fluid was added to each vial and, after $20 \mathrm{~min}$ in the dark, the samples were counted.

For Western blotting, infected cells were harvested at $48 \mathrm{~h}$ p.i., and lysates were prepared as described above. One-hundredth of each supernatant was electrophoresed through SDS-polyacrylamide gels, and the proteins were electrophoretically transferred to a nitrocellulose membrane. The membrane was reacted with anti-GST antibody (Amersham Pharmacia), and bound antibodies were detected with horseradish peroxidase-conjugated rabbit anti-goat immunoglobulin. The signal was detected by chemiluminescence by using an ECL kit (Amersham Pharmacia) according to the manufacturer's instructions.

\section{RESULTS}

GST-UL97 purified from insect cells is partially phosphorylated. Our laboratory previously reported that wt GST-UL97, purified from insect cells after expression by using recombinant baculovirus, becomes phosphorylated on Ser and Thr residues when incubated with $\left[\gamma^{-32} \mathrm{P}\right]$ ATP $(10)$. The phosphorylation rate was first order with protein concentration. Moreover, phosphorylation was essentially eliminated by a point mutation (K355Q) that alters lysine-355 (10), which corresponds to the invariant lysine in subdomain II of protein kinases that is crucial for enzyme activity (8). Thus, UL97 autophosphorylates.

We subsequently observed that wt GST-UL97 and GSTUL97K355Q behaved differently on SDS-PAGE. wt GSTUL97 migrated as a diffuse band (Fig. 1, lane 1), whereas the mutant protein migrated as a single tight band with slightly greater electrophoretic mobility (Fig. 1, lane 3). This suggested that wt GST-UL97 purified from insect cells was modified, most likely by autophosphorylation. To address this possibility, wt GST-UL97 was treated with lambda phosphatase and, indeed, this resulted in the protein migrating similarly to the catalytically inactive mutant protein (Fig. 1, lane 2). This result is similar to the previous report that lambda phosphatase converts the electrophoretic behavior of UL97 from HCMV-infected cells from a broad band to a tight band with greater mobility (24).

To confirm that lambda phosphatase treatment indeed removed phosphates, we incubated either phosphatase-treated or untreated GST-UL97 with $\left[{ }^{32} \mathrm{P}\right] \mathrm{ATP}$ and measured the rate and extent of the incorporation of radioactivity. By $30 \mathrm{~min}$, incorporation reached a maximum (Fig. 2), and the addition of more ATP did not result in further incorporation (data not shown). Slightly more than twice as much phosphate was incorporated into the phosphatase-treated protein ( 7 to $8 \mathrm{~mol}$ of phosphate per mol of GST-UL97) as was incorporated into the untreated protein (3.5 mol of phosphate per mol of GSTUL97, a finding in agreement with previous results [10]). Taken together, these results indicate that wt GST-UL97 purified from insect cells is already partially autophosphorylated.

Phosphorylation of H2B by dephosphorylated and phosphorylated UL97. To examine whether phosphorylation affects the activity of UL97 on an exogenous substrate, we compared the rate and extent of phosphorylation of histone $\mathrm{H} 2 \mathrm{~B}$ by dephosphorylated and phosphorylated GST-UL97. The phosphorylation of H2B can be attributed to UL97, because it is sensitive to the K355Q mutation and to a specific UL97 inhibitor, maribavir (1). As described in Materials and Methods and as presented in a flow chart in Fig. 3, top, GST-UL97 was treated with lambda phosphatase, which resulted in its migrating as a single tight band on SDS-PAGE (Fig. 1, lane 2). The phosphatase activity was inactivated with orthovanadate. Onehalf of the phosphatase-treated GST-UL97 was incubated for $30 \mathrm{~min}$ in kinase buffer with a high concentration of unlabeled ATP to permit efficient autophosphorylation (phosphatasetreated and then autophosphorylated preparation), whereas the other half was incubated similarly in kinase buffer but without ATP (phosphatase-treated preparation). Histone and radiolabeled ATP were then added to both samples, and the phosphatase-treated preparation now received the same high concentration of unlabeled ATP that the phosphatase-treated and then autophosphorylated preparation had previously. If UL97 phosphorylation were required for H2B phosphorylation, then one would predict a lag in the time course of $\mathrm{H} 2 \mathrm{~B}$ phosphorylation by the phosphatase-treated preparation rela-

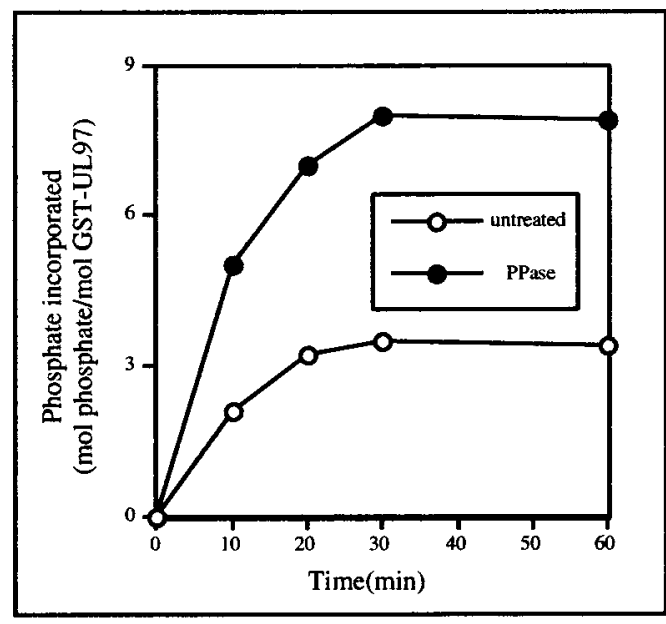

FIG. 2. Stoichiometry of phosphorylation of GST-UL97. A total of $400 \mathrm{ng}$ of either untreated GST-UL97 $(\bigcirc)$ or GST-UL97 that had been treated with lambda phosphatase $(\bullet)$ were incubated in kinase buffer with radiolabeled ATP. Aliquots were removed at the indicated times, and ${ }^{32} \mathrm{P}$ incorporation was measured. 


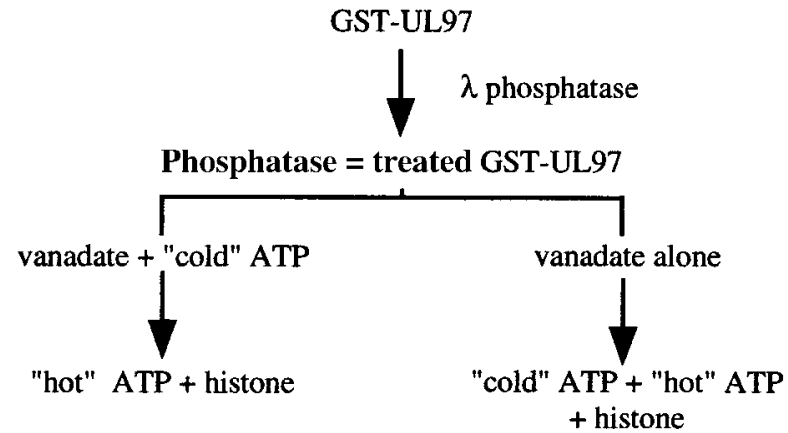

PPase/ATP

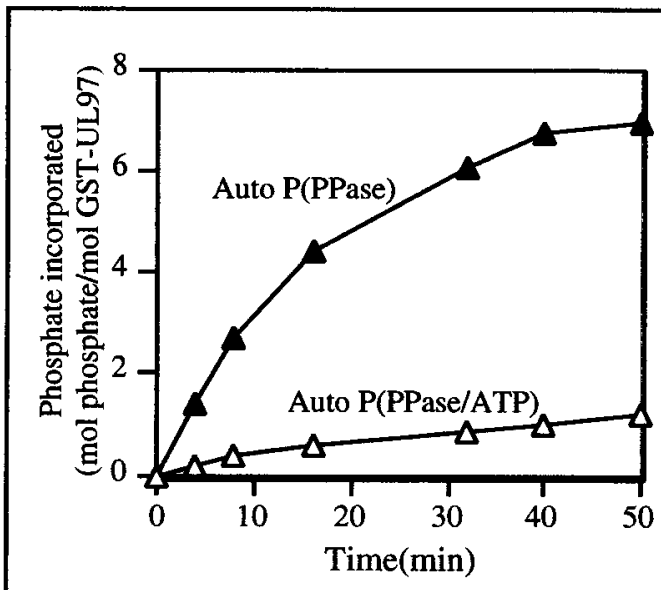

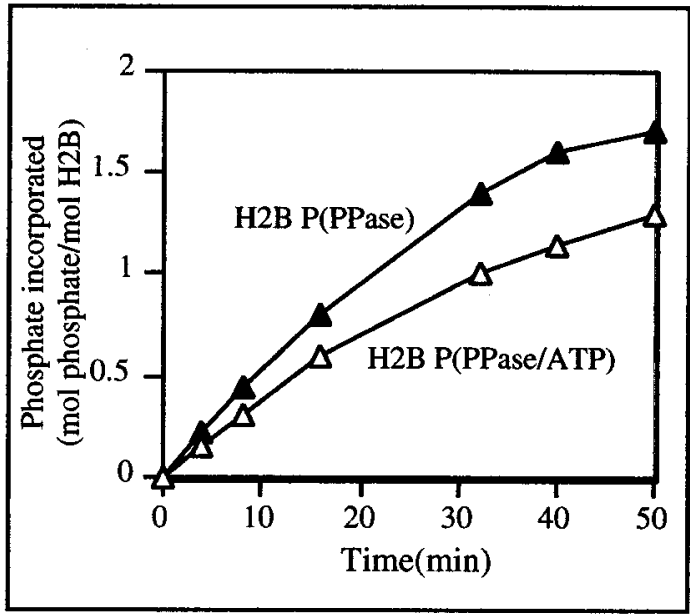

FIG. 3. Effect of lambda phosphatase treatment on rates of autophosphorylation and H2B phosphorylation. (Top) Flow chart of the experiment. (Bottom) Results of the experiment. A total of $800 \mathrm{ng}$ of GST-UL97 was dephosphorylated by lambda ( $\lambda$ ) phosphatase and, as described in Materials and Methods, half was incubated with unlabeled ("cold") ATP for 30 min (PPase/ATP; $\triangle$ ) and the other half was incubated in kinase buffer without ATP (PPase; $\mathbf{\Delta}$ ). Then, $5 \mu \mathrm{g}$ of histone H2B and radiolabeled ("hot") ATP were added to each preparation, with additional unlabeled ("cold") ATP added to the PPase preparation. Aliquots were removed at the indicated times and the amount of phosphate incorporated per mol of GST-UL97 (bottom, left panel; Auto) or histone H2B (bottom, right panel; H2B) were measured.

tive to the phosphatase-treated and then autophosphorylated preparation.

As expected, the phosphatase-treated preparation of UL97 autophosphorylated efficiently (Fig. 3, bottom, left panel). In contrast, the phosphatase-treated and then autophosphorylated preparation of UL97 incorporated relatively little labeled phosphate into itself ( $<1 \mathrm{~mol}$ of phosphate per mol of GSTUL97 in $50 \mathrm{~min}$ ). This residual phosphate incorporation was likely due to exchange between labeled ATP and phosphorylated UL97. Despite the differences in phosphorylation state at the time $\mathrm{H} 2 \mathrm{~B}$ was added, there was no lag in the time course of phosphorylation by the phosphatase-treated preparation (Fig. 3 bottom, right panel). Indeed, the rate of $\mathrm{H} 2 \mathrm{~B}$ phosphorylation by the phosphatase-treated preparation was slightly increased. Thus, there appears to be no requirement that UL97 be phosphorylated for it to phosphorylate an exogenous substrate, $\mathrm{H} 2 \mathrm{~B}$.

Identification of phosphorylation sites in UL97. Previous phosphoamino acid analysis of autophosphorylated UL97 indicated that ca. $65 \%$ of radioactivity comigrated with phospho- serine, and ca. $35 \%$ comigrated with phosphothreonine (10). To identify the specific sites of phosphorylation, we subjected either untreated or phosphatase-treated GST-UL97 to autophosphorylation with $\left[{ }^{32} \mathrm{P}\right] \mathrm{ATP}$, digested the labeled protein with trypsin, and resolved the resulting peptides through a $30 \%$ alkaline polyacrylamide gel to enrich for phosphorylated species. As shown in Fig. 4, both preparations yielded the same 6 major bands, which contained $>95 \%$ of the radioactivity. These bands were named UL97-1 to UL97-6 in order of mobility. UL97-1, -2, and -4 were less intensely labeled and UL97-5 was more intensely labeled in the untreated preparation. Each of the six bands was excised, eluted, and further purified by RP-HPLC. Single major peaks of radioactivity were detected for each band (Table 1). Material from each radioactive RP-HPLC fraction was then subjected to automated peptide sequencing with a portion of the eluant from each sequencing cycle collected and assayed for radioactivity to determine which residues were phosphorylated (Fig. 5).

All sites of phosphorylation identified were in the long $\mathrm{N}$ terminal region of UL97, distant from conserved protein ki- 


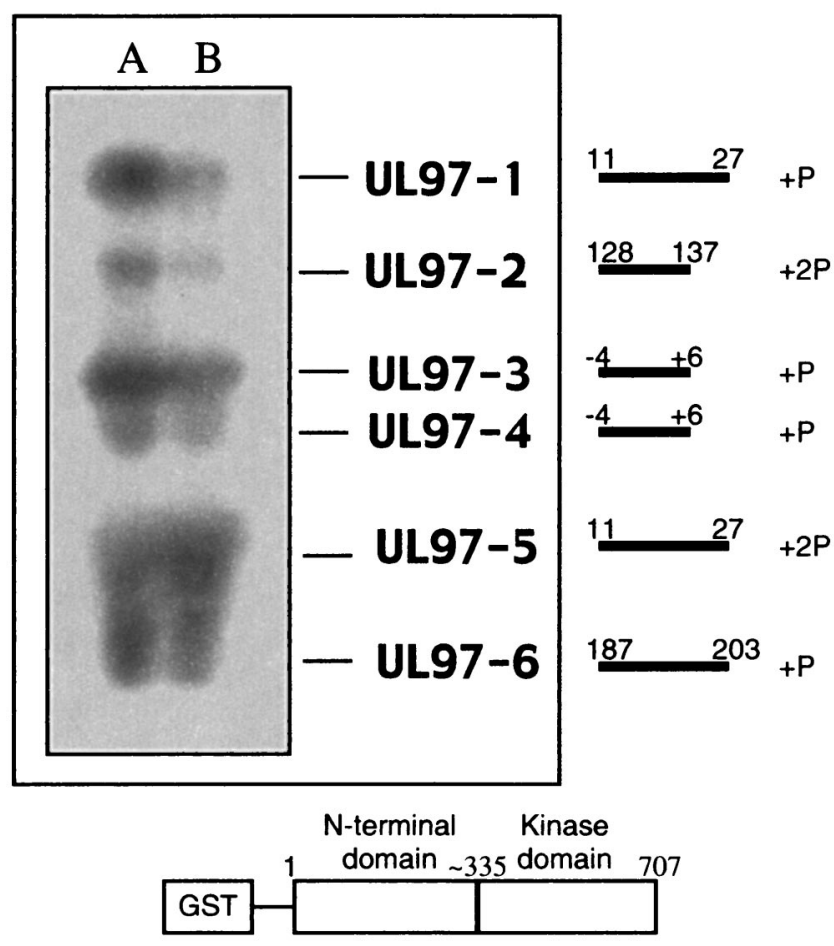

FIG. 4. Tryptic phosphopeptides of GST-UL97. Phosphatasetreated GST-UL97 (A) and untreated GST-UL97 (B) were autophosphorylated with radiolabeled ATP and digested with trypsin. The resulting peptides were resolved on a $30 \%$ (wt/vol) alkaline acrylamide gel. After electrophoresis, the gel was dried onto filter paper and autoradiographed. The positions of the six major species (UL97-1 to UL97-6) are indicated immediately to the right of the autoradiogram. To the right of that are lines and numbers indicating the residues of UL97 that are represented in the various autophosphorylated peptides and the number of phosphate groups $(\mathrm{P})$ in each peptide. Negative numbers refer to residues upstream of UL97 in the GST moiety. Below is a schematic representation of GST-UL97 showing the relative locations of the $\mathrm{N}$-terminal and kinase domains.

nase motifs (Fig. 4). Five Ser residues and four Thr residues were identified as sites of phosphate incorporation in four different segments of UL97 (Fig. 4 and 5): Ser-2 and Ser-3 (peptides UL97-3 and UL97-4); Ser-11, Ser-13, Thr-16, and Thr-18 (UL97-1 and UL97-5); Ser-133 and Thr-134 (UL97-2); and Thr-190 (UL97-6). MALDI-TOF mass spectrometry was performed on material from the radioactive RP-HPLC frac- tions (Table 1), which confirmed the sequence analysis. Mass spectrometry did not reveal any difference that could explain the electrophoretic mobility difference between UL97-3 and UL97-4. However, it did reveal that UL97-1 contained a monophosphorylated species and UL97-5 a diphosphorylated species. Presumably, after phosphatase treatment, autophosphorylation is more likely to add just one phosphate to this segment, resulting in intensely labeled UL97-1. In the absence of phosphatase treatment, one of the four Ser and Thr residues in the segment corresponding to UL97-1 is already phosphorylated. Thus, further autophosphorylation converts this segment into the diphosphorylated form, UL97-5, which is thus more intensely labeled than UL97-1 in the absence of phosphatase treatment.

Since there were nine phosphorylated residues identified in the sequencing analysis (Fig. 5) and seven to eight phosphates incorporated per mole of phosphatase-treated UL97 (Fig. 2 and 3), we infer that some sites were phosphorylated less extensively than others. This was borne out by comparisons of the extent of autophosphorylation of the different segments (Table 1). The N-terminal segment containing Ser-2 and Ser-3 was the most extensively phosphorylated. About $70 \%$ of the radioactivity was incorporated at Ser residues and 30\% at Thr residues, which is in good agreement with the values obtained from phosphoamino acid analysis (10).

Phosphorylation of a synthetic peptide corresponding to the N-terminal autophosphorylation site. The autophosphorylated segment of GST-UL97 containing Ser-2 and Ser-3 is closely linked to GST, which could conceivably contribute sequences that might permit autophosphorylation that would not occur otherwise. To determine whether N-terminal UL97 sequences alone would be sufficient to direct phosphorylation of these sites, a nine-residue peptide, SP-1, corresponding to the extreme $\mathrm{N}$ terminus of UL97 was synthesized (Fig. 6). We tested this peptide as a substrate for UL97 and analyzed its phosphorylation by using a chimeric gel system that permits resolution of both GST-UL97 and the peptide. SP-1 containing Ser-2 and Ser-3 was readily phosphorylated by GST-UL97 (Fig. 6, lane 1). Phosphorylation was eliminated by the K355Q substitution of UL97 (lane 2) and by maribavir (lane 3), which is a specific inhibitor of UL97 $(1,2)$, confirming that phosphorylation was due to UL97 rather than a contaminant. Thus, phosphorylation of this segment does not require the sequences from the GST fusion partner.

SP-1 contains three Ser residues corresponding to Ser-2 and

TABLE 1. Summary of HPLC from each peptide band and mass spectrometric analysis of radioactive HPLC fractions

\begin{tabular}{|c|c|c|c|c|}
\hline Band & $\begin{array}{l}\text { HPLC fraction } \\
\qquad(\min )\end{array}$ & $\begin{array}{l}\text { Radioactivity }^{a} \\
\text { (cpm) }\end{array}$ & $\operatorname{Mass}^{b}(\mathrm{Da})$ & Predicted peptide from GST-UL97 sequence ${ }^{c}$ \\
\hline UL97-1 & 34 & 22,000 & $1,864.4$ & ${ }^{11}$ SASLGTTTQGWDPPLR ${ }^{27}+$ phosphate \\
\hline UL97-2 & 30 & 8,000 & $1,245.8$ & ${ }^{128}$ RPVVPSTSSR $^{137}+$ diphosphate \\
\hline UL97-3 & 29 & 25,000 & $1,116.7$ & ${ }^{-4}$ TVATMSSALR $^{6}+$ phosphate \\
\hline UL97-4 & 30 & 12,000 & $1,117.0$ & ${ }^{-4}$ TVATMSSALR $^{6}+$ phosphate \\
\hline UL97-5 & 34 & 9,000 & $1,944.8$ & ${ }^{11}$ SASLGTTTQGWDPPLR ${ }^{27}+$ diphosphate \\
\hline UL97-6 & 32 & 12,000 & $1,732.6$ & ${ }^{187}{ }^{A L F T G G S D P S D S V S G V R ~}{ }^{203}+$ phosphate \\
\hline
\end{tabular}

${ }^{a}$ Cerenkov counts from each radioactive HPLC fraction.

${ }^{b}$ Masses for peaks determined by MALDI-TOF mass spectrometry.

${ }^{c}$ The tryptic fragments of GST-UL97 derived from peptide masses by using program MS-Digest (ProteinProspector V3.2.1) available online at (http:/prospector.ucsf.edu). Numbers above the first and last residues of each phosphopeptide indicate their position within the UL97 sequence. Negative numbers refer to residues upstream of UL97 in the fusion protein. 

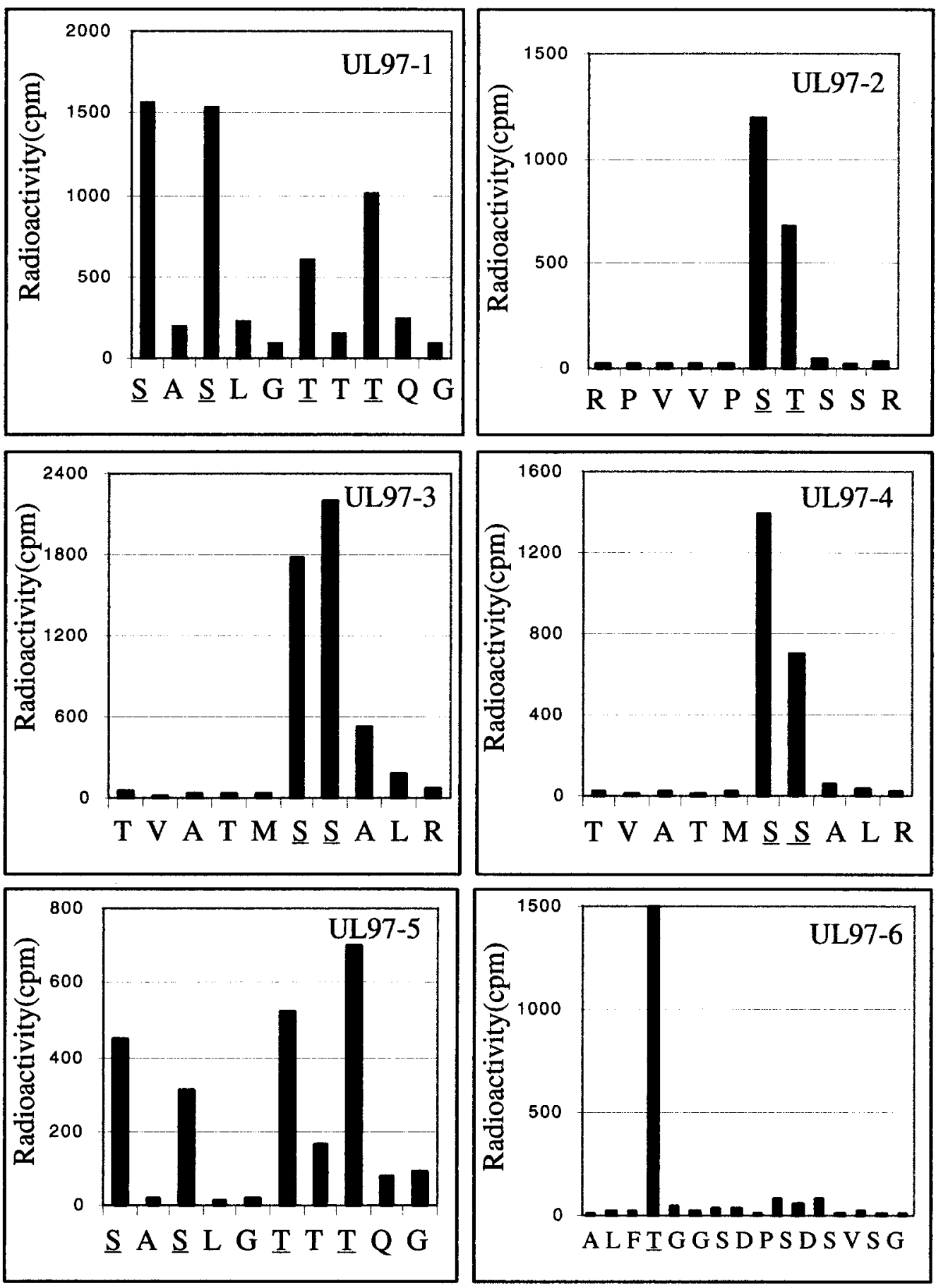

FIG. 5. Sequence analysis of UL97 phosphopeptides. Tryptic phosphopeptides UL97-1 to UL97-6 (as indicated at the top of each panel) were further purified by RP-HPLC, and peak fractions were subjected to automated amino-terminal microsequencing. The material released at each cycle was split to identify the amino acid by RP-HPLC (shown at the bottom of each panel) and the radioactivity by scintillation counting (indicated by black bars). Phosphorylated residues are underlined.

Ser-3, both of which were autophosphorylated by GST-UL97 when present in the whole protein, and Ser-7, which was not detectably autophosphorylated. To determine which Ser residue(s) were phosphorylated in the peptide, SP-1 phosphopeptide was extracted from a gel and analyzed by amino acid sequencing (Fig. 7). This analysis revealed that phosphoryla- tion occurred predominantly at Ser-3. Ser-7 was phosphorylated much less extensively and Ser-2 was not detectably phosphorylated.

Effects of deletion of $\mathrm{N}$-terminal residues on autophosphorylation and H2B phosphorylation. To further test the importance of autophosphorylation for the activities of UL97 against 


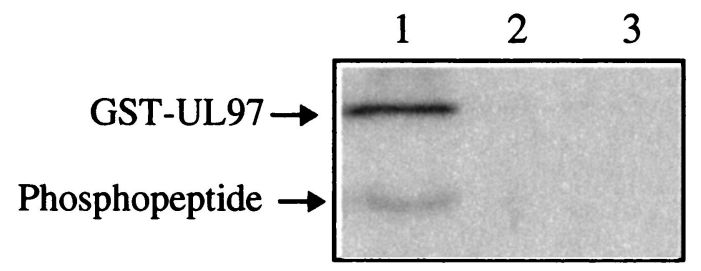

FIG. 6. Phosphorylation of SP-1 by GST-UL97. Synthetic peptide SP-1 $(200 \mu \mathrm{M})$ was phosphorylated in kinase buffer for $30 \mathrm{~min}$ by using $200 \mathrm{ng}$ of GST-UL97 (lanes 1 and 3) or GST-UL97K355Q (lane 2), either in the presence (lane 3 ) or absence of $3 \mu \mathrm{M}$ maribavir (lanes 1 and 2). The reaction products were resolved by electrophoresis on a chimeric polyacrylamide gel, which was analyzed by a phosphorimager. The locations of GST-UL97 and phosphopeptide are indicated to the left.

exogenous substrates, we constructed two baculovirus recombinants each of which expresses a truncated form of GSTUL97 that lacks the N-terminal region containing the autophosphorylation sites mapped above. These mutants, $\Delta$ N239 and $\Delta$ N277 are missing the first 239 and 277 amino acids of UL97, respectively, but they retain all of the UL97 sequences that align with the conserved motifs of known protein kinases. The truncated proteins were purified and tested for autophosphorylation and for histone phosphorylation, by using equivalent molar amounts of each UL97 derivative (Fig. 8). Based on phosphorimager analysis of the gel shown in Fig. 8A, autophosphorylation by $\Delta \mathrm{N} 239$ was reduced 20 -fold relative to wt GST-UL97, whereas autophosphorylation by $\Delta$ N277 was reduced 100 -fold. It is possible that the low level of autophosphorylation of these mutants is due to a very minor phosphorylation site not detected in our analysis of wt GST-UL97 or to a new site recognized by mutant, but not wt protein. Either way, making the conservative assumption that there is only one phosphorylation site remaining per mutant protein, only a tiny fraction of these mutant proteins would be phosphorylated.

Despite their drastically reduced autophosphorylation, both $\Delta \mathrm{N} 239$ and $\Delta \mathrm{N} 277$ exhibited substantial phosphorylation of histone H2B (Fig. 8A). Phosphorimager analysis of the gel

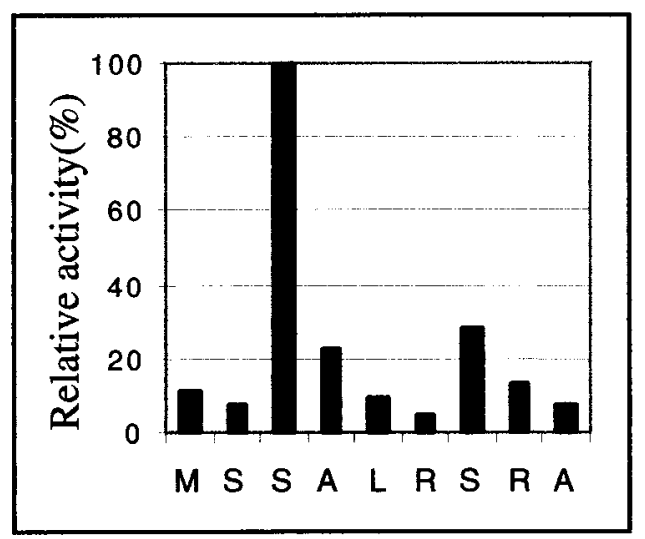

FIG. 7. Sequence analysis of phosphorylated SP-1. After phosphorylation of SP-1 by GST-UL97, the radiolabeled peptide was resolved from GST-UL97 by gel electrophoresis, extracted from the gel, and analyzed by amino acid sequencing as described in the legend to Fig. 5 , with the radioactivity at cycle 3 set to $100 \%$.

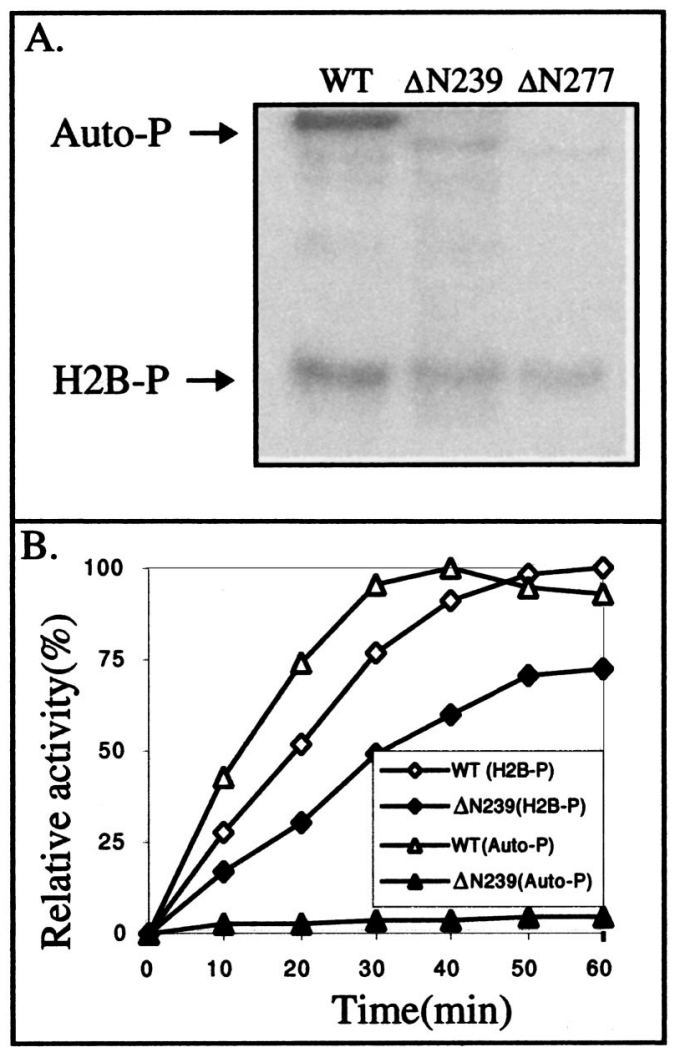

FIG. 8. Differential effects of N-terminal truncations on autophosphorylation and H2B phosphorylation. Equivalent amounts of wt GSTUL97 or N-terminal truncation mutants, $\Delta$ N239 and $\Delta$ N277, were incubated with histone $\mathrm{H} 2 \mathrm{~B}$ and radiolabeled ATP in kinase buffer. At various times, aliquots were analyzed for autophosphorylation or $\mathrm{H} 2 \mathrm{~B}$ phosphorylation. (A) Phosphorimage of an SDS-polyacrylamide gel of radiolabeled products after $30 \mathrm{~min}$ of incubation. The GST-UL97 proteins are indicated at the top of the panel, and the positions of autophosphorylated GST-UL97 (Auto-P) and phosphorylated H2B (H2B-P) are indicated to the left. (B) Time course of the reactions for wt GST-UL97 (WT; open symbols) and $\Delta$ N239 (solid symbols) for autophosphorylation (Auto-P; triangles) and $\mathrm{H} 2 \mathrm{~B}$ phosphorylation (H2B-P; diamonds). The maximal incorporation for autophosphorylation and $\mathrm{H} 2 \mathrm{~B}$ phosphorylation by wt enzyme was set as $100 \%$.

indicated that $\mathrm{H} 2 \mathrm{~B}$ phosphorylation was ca. 70 and $50 \%$ that of wt GST-UL97, for $\Delta \mathrm{N} 239$ and $\Delta \mathrm{N} 277$, respectively, after 30 min of incubation. To examine this more closely, the rates of autophosphorylation and $\mathrm{H} 2 \mathrm{~B}$ phosphorylation by wt GSTUL97 and $\Delta$ N239 were compared in a time course study (Fig. $8 \mathrm{~B}$ ). The rate of $\mathrm{H} 2 \mathrm{~B}$ phosphorylation by $\Delta \mathrm{N} 239$ was $\sim 70 \%$ that of wt GST-UL97. Thus, a mutation that effectively eliminates autophosphorylation by UL97 has only minor effects on the ability of UL97 to phosphorylate H2B.

Effects of deletion of N-terminal residues on GCV anabolism. GCV is a very weak substrate for UL97 (23; unpublished results), making it difficult to compare GCV phosphorylation by mutant proteins in vitro. We therefore compared GCV phosphorylation in insect cells, directed by wt and mutant GST-UL97s expressed by recombinant baculoviruses, by using an approach similar to one previously described (10) The mutant proteins tested were the two deletion mutants described above; a third deletion mutant $\Delta \mathrm{N} 303$, which removes an even 


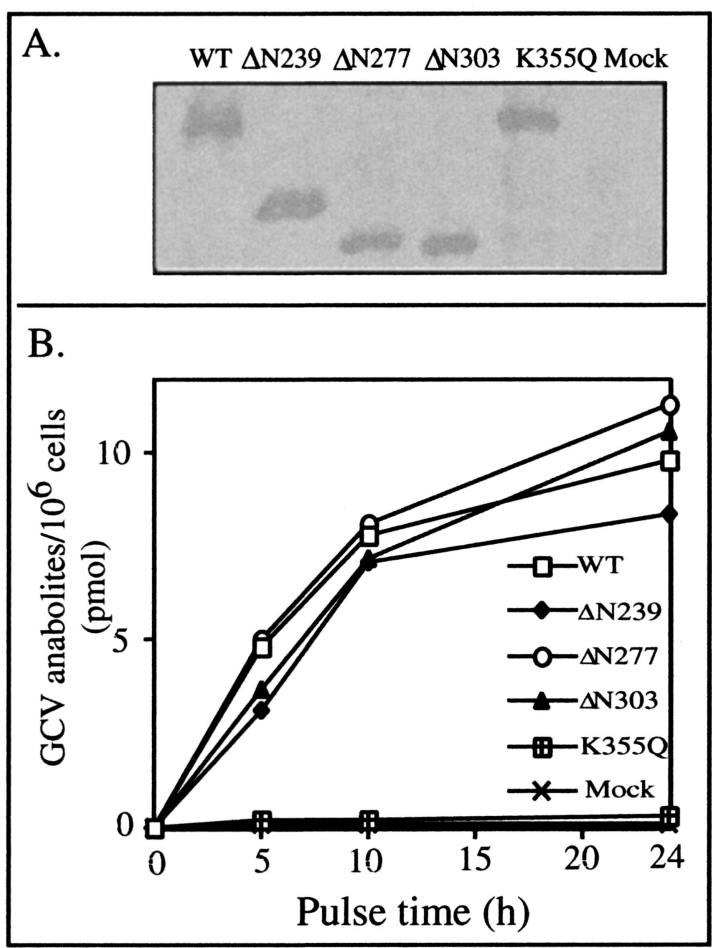

FIG. 9. Large N-terminal truncations of UL97 do not decrease GCV anabolism. Insect cells were mock infected or infected with recombinant baculoviruses expressing the indicated GST-UL97 proteins. (A) Lysates were prepared at $48 \mathrm{~h}$ p.i., and proteins were resolved by SDS-PAGE and transferred to nitrocellulose. The nitrocellulose was reacted with an anti-GST antibody, which was detected with horseradish peroxidase-conjugated rabbit anti-goat immunoglobulin antibody. The versions of GST-UL97 expressed in each lysate are indicated at the top of the panel. (B) At $24 \mathrm{~h}$ p.i., cells were pulsed with radiolabeled GCV for the times indicated, lysates were prepared, and radiolabled GCV anabolites were measured by using a filter-binding assay. WT, wild type; Mock, mock infected.

larger portion of the N-terminal region of UL97, and GSTUL97K355Q, which encodes full-length, but catalytically inactive UL97. (The mutant protein expressed by $\Delta$ N303 was difficult to purify in active form and was not included in the biochemical study described above.) At the multiplicities of infection used, each baculovirus expressed similar amounts of GST-UL97, as determined by Western blotting (Fig. 9A). The baculovirus expressing wt GST-UL97 and the three baculoviruses expressing N-terminally truncated forms of UL97 directed similar amounts of GCV phosphorylation over a range of pulse times ranging from 5 to $24 \mathrm{~h}$, whereas the virus expressing the catalytically inactive mutant, K355Q, directed little or no GCV phosphorylation above that in mock-infected cells (Fig. 9B). Thus, mutants that lack autophosphorylation sites, and are thus highly impaired for autophosphorylation, are not meaningfully impaired for phosphorylation of GCV in insect cells.

\section{DISCUSSION}

We took two approaches to examine whether autophosphorylation of UL97 affects the activity of this kinase on exogenous substrates. The first approach, which was biochemical, showed that neither dephosphorylation nor extensive autophosphorylation had any major effect on the ability of GST-UL97 to phosphorylate an exogenous protein substrate, histone $\mathrm{H} 2 \mathrm{~B}$. The second approach was made possible by our mapping of the autophosphorylation sites of UL97 to within the first 177 residues of the protein. We constructed mutant forms of UL97 that lacked these residues and, accordingly, were highly impaired for autophosphorylation. These mutants were still able to phosphorylate $\mathrm{H} 2 \mathrm{~B}$ in vitro and $\mathrm{GCV}$ in insect cells. We therefore conclude that autophosphorylation of UL97 is not required for its activity on exogenous substrates.

This finding contrasts with that reached by another group of investigators (14), who concluded that phosphorylation of UL97 is essential for GCV phosphorylation. There are several technical differences between the two studies. Autophosphorylation was assayed using purified baculovirus-expressed GST fusion protein in our study and by using nuclear matrix fractions containing vaccinia virus-expressed UL97 in the other study. GCV phosphorylation was measured in insect Sf9 cells in our study and in human 143 cells in the other by somewhat different assay techniques. It is conceivable that the different conclusions reached by the two studies are due to these technical differences. (Also, there is no guarantee that the locations of autophosphorylation sites and the effects of mutations on heterologously expressed UL97 would be the same on UL97 expressed in HCMV-infected cells. It would, however, be extremely difficult to perform the kinds of studies described herein with enzyme from HCMV-infected cells.) Regardless, our study perturbed the phosphorylation state of UL97 specifically, either enzymatically or by mutations that removed the autophosphorylation sites. The other study employed mutations that alter conserved motifs that might be expected to impair protein kinase activity generally. These mutations are akin to the K355Q mutation in conserved subdomain II that we have shown inactivates autophosphorylation (9), H2B phosphorylation (1), and acyclovir and GCV phosphorylation (23; this study). We suggest that such mutations inactivate the catalytic activity of the enzyme generally rather than specifically impair autophosphoryation.

The ability of deletion mutants $\Delta \mathrm{N} 239, \Delta \mathrm{N} 277$, and $\Delta \mathrm{N} 303$ to direct GCV phosphorylation in insect cells is consistent with a previous study which showed that UL97 deletion mutants lacking segments of the N-terminal domain could direct GCV phosphorylation in recombinant vaccinia virus-infected 143 cells (16). Although one of these mutants (described variously as lacking 240 or 292 residues) exhibited reduced GCV phosphorylation, expression of this protein was also much reduced. That mutant protein also exhibited severely reduced phosphorylation after incubation of a crude nuclear matrix fraction with radiolabeled ATP. That result might be consistent with our mapping of autophosphorylation sites but might also have been due to low expression of UL97. In the same study, a pair of mutants lacking the $\mathrm{N}$-terminal 110 residues were phosphorylated after incubation with radiolabeled ATP, which led the authors to conclude that the N-terminal 110 residues of UL97 have no influence on phosphorylation of UL97. This conclusion contrasts with our findings showing that major sites of autophosphorylation lie within these residues; however, the previous study neither used purified protein nor a quantitative assay of autophosphorylation. 
To our knowledge, UL97 is the first herpesvirus protein kinase with clear homology to conventional protein kinases for which autophosphorylation sites have been identified. The most extensively autophosphorylated segment was near the extreme $\mathrm{N}$ terminus of UL97. Although our studies were performed with a GST fusion protein, we infer that this segment would be autophosphorylated with native protein, as a peptide, SP-1, corresponding to the $\mathrm{N}$ terminus is a robust substrate for UL97. It may be, however, that the presence of residues from the fusion construct can influence the pattern of phosphorylation as both Ser-2 and Ser-3 were extensively autophosphorylated in the context of GST-UL97, but only Ser-3 was extensively phosphorylated in the synthetic peptide. Also, Ser-7 was phosphorylated to a modest extent in the synthetic peptide. This limited phosphorylation and its location in a two-residue tryptic peptide may explain why autophosphorylation of this residue was not detected in the context of GST-UL97.

The robust phosphorylation of SP-1 sheds light on the substrate specificity of UL97. SP-1 lacks residues $\geq 3$ positions $\mathrm{N}$-terminal to the site of phosphorylation (P-3 and beyond). The $\mathrm{P}-3$ position is crucial for substrate recognition by many protein kinases (21) but evidently is not essential for UL97. Like other peptide substrates strongly phosphorylated by UL97 (1), SP-1 contains a basic residue at the $\mathrm{P}+5$ position. We have recently found that this position is crucial for substrate recognition by UL97 (1) and speculate that the basic character of this position is important.

None of the autophosphorylation sites lies within the Cterminal domain of UL97 that contains conserved protein kinase motifs. This finding is consistent with our failure to detect any major effects of either phosphatase treatment or deletion of these sites on catalytic activity. Although phosphatase treatment appeared to slightly increase $\mathrm{H} 2 \mathrm{~B}$ phosphorylation, deletion of segments, including autophosphorylation sites, slightly decreased phosphorylation. Thus, we cannot exclude the possibility that autophosphorylation slightly affects UL97 protein kinase activity either positively or negatively.

There is evidence that UL97 plays a role during HCMV replication in viral DNA replication, DNA encapsidation, and/or nuclear egress $(2,11 \mathrm{a}, 26)$, all of which occur in the nucleus. If UL97 participates directly in any of these processes, its nuclear localization should be important. It has been reported that residues 1 to 110 are necessary for efficient nuclear localization of UL97 and are sufficient to direct nuclear localization of green fluorescent protein $(15,16)$. Since this segment contains six of the nine autophosphorylation sites, it is possible that autophosphorylation could modulate nuclear localization, by effects on import and/or export, as is the case for yeast Pho4 (11). Alternatively, autophosphorylation could be involved in protein-protein interactions that, for example, aid substrate recognition or incorporation into virions. Our identification of mutants that are highly impaired for autophosphorylation, but not for phosphorylation of exogenous substrates, should greatly aid studies to determine the role, if any, of autophosphorylation in HCMV replication.

\section{ACKNOWLEDGMENTS}

We gratefully acknowledge Z. He for initial work on this project, including the construction of baculovirus recombinants expressing truncated forms of UL97; A. Dickerson and J. Lee of the MBCF of the
Dana-Farber Cancer Insititute for amino acid, sequencing, and mass spectrometric analyses; and C. Talarico and K. Biron of Glaxo SmithKline for generous provision of GCV and maribavir and for communicating results prior to publication.

This work was supported in part by grant RO1 AI26077 (to D.M.C.) from the National Institutes of Health. M.-C.B. was supported in part by the Korean Science and Engineering Foundation. P.M.K. was supported in part by training grant T32 AI07245 from the National Institutes of Health.

\section{REFERENCES}

1. Baek, M.-C., P. M. Krosky, Z. He, and D. M. Coen. 2002. Specific phosphorylation of exogenous protein and peptide substrates by the human cytomegalovirus UL97 protein kinase: importance of the $\mathrm{P}+5$ position. J. Biol. Chem. 277:29593-29599.

2. Biron, K. K., R. J. Harvey, S. C. Chamberlain, S. S. Good, A. A. Smith III, M. G. Davis, C. L. Talarico, W. H. Miller, R. Ferris, R. E. Dornsife, S. C. Stanat, J. C. Drach, L. B. Townsend, and G. W. Koszalka. 2002. Potent and selective inhibition of human cytomegalovirus replication by 1263 W94, a benzimidazole L-riboside with a unique mode of action. Antimicrob. Agents Chemother. 46:2365-2372.

3. Chee, M. S., G. L. Lawrence, and B. G. Barrell. 1989. Alpha-, beta-, and gammaherpesviruses encode a putative phosphotransferase. J. Gen. Virol. 70:1151-1160.

4. Crossen, R., and S. Gruenwald. 1998. Baculovirus expression vector system manual, 5th ed. Pharmingen, San Diego, Calif.

5. Dadd, C. A., R. G. Cook, and C. D. Allis. 1993. Fractionation of small tryptic phosphopeptides by alkaline PAGE followed by amino acid sequencing. BioTechniques 14:266-273.

6. de Wind, N., J. Domen, and A. Berns. 1992. Herpesviruses encode an unusual protein-serine/threonine kinase which is nonessential for growth in cultured cells. J. Virol. 66:5200-5209.

7. Drew, W. L., and W. C. Buhles. 1996. Cytomegalovirus: nucleosides and foscarnet: clinical applications, p. 153-166. In D. D. Richman (ed.), Antiviral drug resistance. John Wiley \& Sons, Chichester, England.

8. Hanks, S. K., and T. Hunter. 1995. The eukaryotic protein kinase superfamily: kinase (catalytic) domain structure and classification. FASEB J. 9:576596.

9. Hardie, D. G. 1995. Cellular functions of protein kinases, p. 48-56. In D. G. Hardie and S. Hanks (ed.), The protein kinase facts book, vol. 1. Academic Press, London, England.

10. He, Z., Y.-S. He, Y. Kim, L. Chu, C. Ohmstede, K. K. Biron, and D. M. Coen. 1997. The human cytomegalovirus UL97 protein is a protein kinase that phosphorylates on serines and threonines. J. Virol. 71:405-411.

11. Komeili, A., and E. K. O'Shea. 1999. Roles of phosphorylation sites in regulating activity of the transcription factor Pho4. Science 284:977-980.

11a.Krosky, P. M., M.-C. Baek, and D. M. Coen. The human cytomegalovirus UL97 kinase, an antiviral drug target, is required at the stage of nuclear egress. J. Virol., in press.

12. Littler, E., A. D. Stuart, and M. S. Chee. 1992. Human cytomegalovirus UL97 open reading frame encodes a protein that phosphorylates the antiviral nucleoside analogue ganciclovir. Nature 358:160-162.

13. McGeoch, D. J., L. J. Coulter, and H. W. M. Moss. 1995. U protein kinases (herpesviruses), p. 391-393. In D. G. Hardie and S. Hanks (ed.), The protein kinase facts book, vol. 1. Academic Press, London, England.

14. Michel, D., S. Kramer, S. Höhn, P. Schaarschmmidt, K. Wunderlich, and T. Mertens. 1999. Amino acids of conserved kinase motifs of cytomegalovirus protein UL97 are essential for autophosphorylation. J. Virol. 73:88988901.

15. Michel, D., I. Pavic, A. Zimmermann, E. Haupt, K. Wunderlich, M. Heuschmid, and T. Mertens. 1996. The UL97 gene product of human cytomegalovirus is an early-late protein with a nuclear localization but is not a nucleoside kinase. J. Virol. 70:6340-6346.

16. Michel, D., P. Schaarschmidt, K. Wunderlich, M. Heuschmid, L. Simoncini, D. Mühlberger, A. Zimmermann, I. Pavic, and T. Mertens. 1998. Functional regions of the human cytomegalovirus protein pUL97 involved in nuclear localization and phosphorylation of ganciclovir and pUL97 itself. J. Gen. Virol. 79:2105-2112.

17. Mizzen, C. A., Y. Dou, Y. Liu, R. G. Cook, M. A. Gorovsky, and C. D. Allis. 1999. Identification and mutation of phosphorylation sites in a linker histone. J. Biol. Chem. 274:14533-14536.

18. Prichard, M. N., N. Gao, S. Jairath, G. Mulamba, P. Krosky, D. M. Coen, B. O. Parker, and G. S. Pari. 1999. A recombinant human cytomegalovirus with a large deletion in UL97 has a severe replication deficiency. J. Virol. 73:5663-5670.

19. Shevchenko, A., M. Wilm O. Vorm, and M. Mann. 1996. Mass spectrometric sequencing of proteins from silver-stained polyacrylamide gels. Anal. Chem. 68:850-858.

20. Smith, R. F., and T. F. Smith. 1989. Identification of new protein-kinase related genes in three herpesviruses, herpes simplex virus, varicella zoster virus, and Epstein-Barr virus. J. Virol. 63:450-455. 
21. Songyang, Z., K. P. Lu, Y. T. Kwon, L.-H. Tsai, O. Filhol, C. Cochet, D. A Brickey, T. R. Soderling, C. Bartleson, D. J. Graves, A. J. Demaggio, M. F Hoekstra, J. Blenis, T. Hunter, and L. C. Cantley. 1996. A structural basis for substrate specificities of protein Ser/Thr kinases: primary sequence preference of casein kinases I and II, NAMA, phosphorylase kinase, calmodulindependent kinase II, CDK5, and Erk1. Mol. Cell. Biol. 16:6486-6493.

22. Sullivan, V., C. L. Talarico, S. C. Stanat, M. Davis, D. M. Coen, and K. K. Biron. 1992. A protein kinase homologue controls phosphorylation of ganciclovir in human cytomegalovirus-infected cells. Nature 358:162-164.

23. Talarico, C. L., T. C. Burnette, W. H. Miller, S. L. Smith, M. G. Davis, S. C. Stanat, T. I. Ng, Z. He, D. M. Coen, B. Roizman, and K. K. Biron. 1999.
Acyclovir is phosphorylated by the human cytomegalovirus UL97 protein. Antimicrob. Agents Chemother. 43:1941-1946.

24. van Zeijl, M., J. Fairhurst, E. Z. Baum, L. Sun, and T. R. Jones. 1997. The human cytomegalovirus UL97 protein is phosphorylated and a component of virions. Virology 231:72-80.

25. West, M. H. P., R. S. Wu, and W. M. Bonner. 1984. Polyacrylamide gel electrophoresis of small peptides. Electrophoresis 5:133-138.

26. Wolf, D. G., C. T. Courcelle, M. N. Prichard, and E. S. Mocarski. 2001 Distinct and separate roles for herpesvirus-conserved UL97 kinase in cytomegalovirus DNA synthesis and encapsidation. Proc. Natl. Acad. Sci. USA 98:1895-1900. 